\title{
Thermally Enhanced Neutralization in Hyperthermal Energy Ion Scattering
}

\author{
C.E. Sosolik,f J.R. Hampton, A.C. Lavery, and B.H. Cooperf \\ Laboratory of Atomic and Solid State Physics, \\ Cornell University, Ithaca, New York 14853-2501 \\ J.B. Marston \\ Department of Physics, Box 1843, Brown University, Providence, Rhode Island 02912
}

(Dated: February 7, 2020)

\begin{abstract}
Neutralization probabilities are presented for hyperthermal energy $\mathrm{Na}^{+}$ions scattered from a $\mathrm{Cu}(001)$ crystal as a function of surface temperature and scattered velocity. A large enhancement in neutralization is observed as the temperature is increased. Velocity-dependent charge transfer regimes are probed by varying the incident energy, with the most prominent surface temperature effects occurring at the lowest energies. The data agree well with results obtained from a model based on the Newns-Anderson Hamiltonian, where the effects of both temperature and velocity are incorporated.
\end{abstract}

PACS numbers: 34.50.Dy, 34.70.+e, 79.20.Rf 
Measurements of the charge states of alkali ions scattered from metal surfaces at hyperthermal energies $(\approx 1 \mathrm{eV}$ to $1 \mathrm{keV})$ have achieved remarkable success in isolating factors that govern neutralization at surfaces [1]. From these studies, it is well known that the magnitude of the neutralization probability, $P_{0}$, depends strongly on the value of the projectile ionization potential, $I_{0}$, and the surface work function, $\Phi$, while the rate of charge transfer is determined by the projectile-surface coupling [2]. The effects of surface temperature or $T_{\mathrm{S}}$ on neutralization at a surface, however, have typically been ignored experimentally. In fact, few quantitative measurements [3, 国 have explored the role of $T_{\mathrm{S}}$ in determining $P_{0}$, despite the many theoretical studies $[5,6,0,8,9,10,11,12,13]$ that have been devoted to the subject.

In this Letter, we present quantitative results which show that $T_{\mathrm{S}}$ has significant effects on the neutralization probability. Our measurements involve scattering $\mathrm{Na}^{+}$ions from a $\mathrm{Cu}(001)$ surface as a function of incident energy and $T_{\mathrm{S}}$. The $\mathrm{Na}-\mathrm{Cu}(001)$ scattering system is interesting because both energy-dominated and coupling-dominated charge transfer regimes are accessible and can be probed by varying the incident energy or scattered velocity [14]. In the energy-dominated regime, the change in $P_{0}$ with scattered velocity is governed primarily by the relative values of $I_{0}$ and $\Phi$. In the coupling-dominated regime, the tunneling of electrons between the projectile and surface or equivalently, the projectilesurface coupling, governs the velocity dependence [14]. Our results show that increasing $T_{\mathrm{S}}$ can change $P_{0}$ in both of these regimes, enhancing it by as much as a factor of three at the lowest incident energies. The effects of $T_{\mathrm{S}}$ and scattered velocity on $P_{0}$ have been incorporated into a quantum mechanical model that treats the electrons involved in the charge transfer as independent particles. Results obtained from this model compare well with the trends seen in our experimental data.

Our measurements were performed in an ultra high vacuum chamber and beamline that are described in detail elsewhere [15, 16]. All of the ion beams were produced in a Colutron ion source that has been modified to allow highly efficient alkali ion beam production from a solid state source [17]. The beams were scattered along the $\langle 100\rangle$ azimuth of a $\mathrm{Cu}(001)$ single crystal sample. Surface cleanliness and long range order were monitored using Auger electron spectroscopy and low energy electron diffraction, respectively. The sample temperature was varied between $200 \mathrm{~K}$ and $1100 \mathrm{~K}$ during scattering using a combination of cooling from a liquid nitrogen reservoir connected by copper braids and heating from an electron-beam 
heater mounted behind the sample.

Velocity-resolved charge state fractions were obtained for particles scattered from the sample using time-of-flight techniques along with a neutral particle detector (NPD) [18]. All measurements were made at incident and final angles of $45^{\circ}$ in the plane defined by the incident beam direction and the surface normal. A typical time-of-arrival spectrum obtained using the NPD is shown in Fig. 11 for $250 \mathrm{eV} \mathrm{Na}^{+}$scattered from the $\mathrm{Cu}(001)$ crystal at a temperature of $328 \mathrm{~K}$. The dashed line shows the signal due to both the neutral atoms and ions, the total flux, and the solid line shows the signal coming from the neutrals only. $P_{0}$ was obtained by integrating the intensity of the total and neutral spectra and taking a ratio of the results [19.

To illustrate the dependence of $P_{0}$ on $T_{\mathrm{S}}$, typical results obtained for incident $\mathrm{Na}^{+}$projectiles at energies of $22 \mathrm{eV}, 152 \mathrm{eV}$, and $640 \mathrm{eV}$ are shown in Fig. 2. There is a significant increase in $P_{0}$ at all incident energies as $T_{\mathrm{S}}$ is increased. At each incident energy, the change in neutralization probability is monotonic with $T_{\mathrm{S}}$, with the largest change occurring at the lowest incident energy.

The dependence of $P_{0}$ on incident energy can also be observed from the data presented in Fig. 2. Above $750 \mathrm{~K}, P_{0}$ decreases monotonically as the incident energy is increased. However, below $750 \mathrm{~K}$, the incident energy dependence is non-monotonic. This can be seen more clearly in Fig. 3, where $P_{0}$ has been plotted as a function of the inverse perpendicular velocity of the scattered projectiles at three different $T_{\mathrm{S}}$ values. The non-monotonic change in neutralization is very apparent at $350 \mathrm{~K}$, the lowest temperature shown.

To understand the velocity- and temperature-dependent results shown in Figs. 2 and 3 we first focus on the energy and time dependence of charge transfer for this system at a fixed temperature. We show that the data collected at $350 \mathrm{~K}$ exhibit aspects of both energy- and coupling-dominated charge transfer. Then we examine the effects of increased temperature and discuss why the effects are so large at the lowest incident energies. Finally, we compare our measurements to the results of a theoretical model that incorporates the effects of velocity and temperature.

The energetics of neutralization in ion-surface scattering experiments are typically described using an ionization level diagram. The ionization level, $I(z)=I_{0}-1 / 4 z$, represents both the bare ionization potential of the projectile and the distance dependence induced by the presence of an image charge in the metal [20]. A level diagram for $\mathrm{Na}\left(I_{0}=5.14 \mathrm{eV}\right)$ 


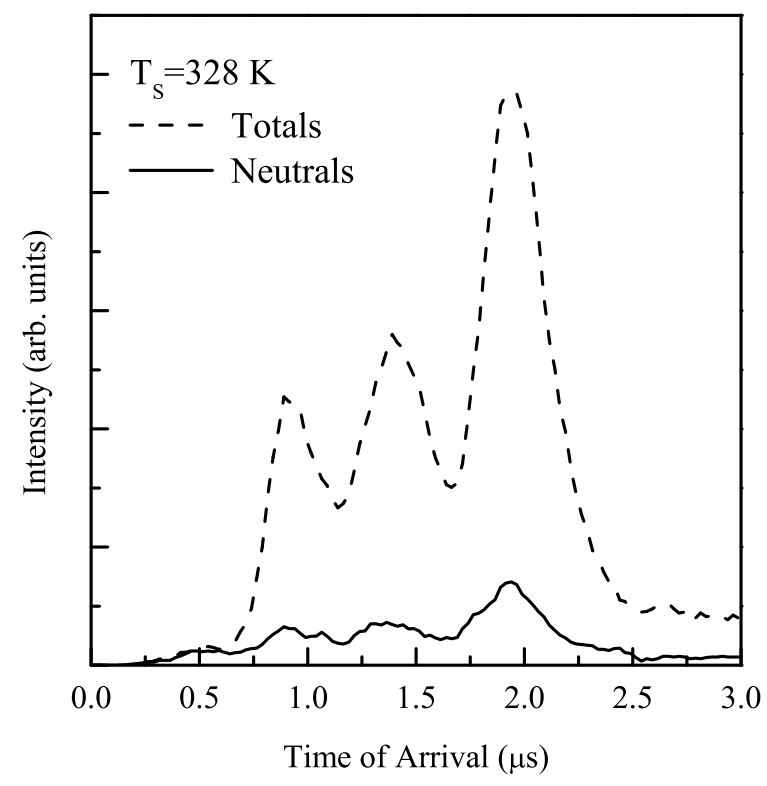

FIG. 1: Time-of-arrival spectra obtained with the NPD for $250 \mathrm{eV} \mathrm{Na}{ }^{+}$ions scattered from a $\mathrm{Cu}(001)$ crystal. The surface temperature was held at $328 \pm 5 \mathrm{~K}$ during this measurement. The three peaks present in these spectra are due to trajectory types that involve scattering from one or more individual atoms at the surface [1].

outside of a $\mathrm{Cu}(001)$ surface is shown in Fig. 1. At any distance $z$ the energetically-favored charge state of the $\mathrm{Na}$ is given by the position of $I(z)$ relative to the $\mathrm{Cu}(001)$ Fermi level, $E_{\mathrm{F}}$. At large distances ( $z>13$ a.u.) the ionization level is below $E_{\mathrm{F}}$, and the energetically-favored charge state is neutral. Closer to the surface, the positive ion is the energetically-favored charge state, as the ionization level is shifted above $E_{\mathrm{F}}$. The level diagram illustrates that different charge states are energetically favored at different $z$ values. This description is incomplete, however, as the projectile-surface coupling must also be considered.

The projectile-surface coupling introduces dynamical and charge state mixing effects that play an important role in determining the final charge state measured in a neutralization experiment. The dynamical effect refers to the competition that arises between the rate of electron tunneling and the finite velocity of the scattered projectile. Put simply, as a projectile leaves the surface, electrons tunnel between the projectile and surface, tracking the energetically favored charge state described above. However, the exponential decay of the coupling with distance implies that at some distance $z$ the charge state is "frozen in" and the projectile is left as either neutral or positive. Quantum-mechanical mixing of projectile 


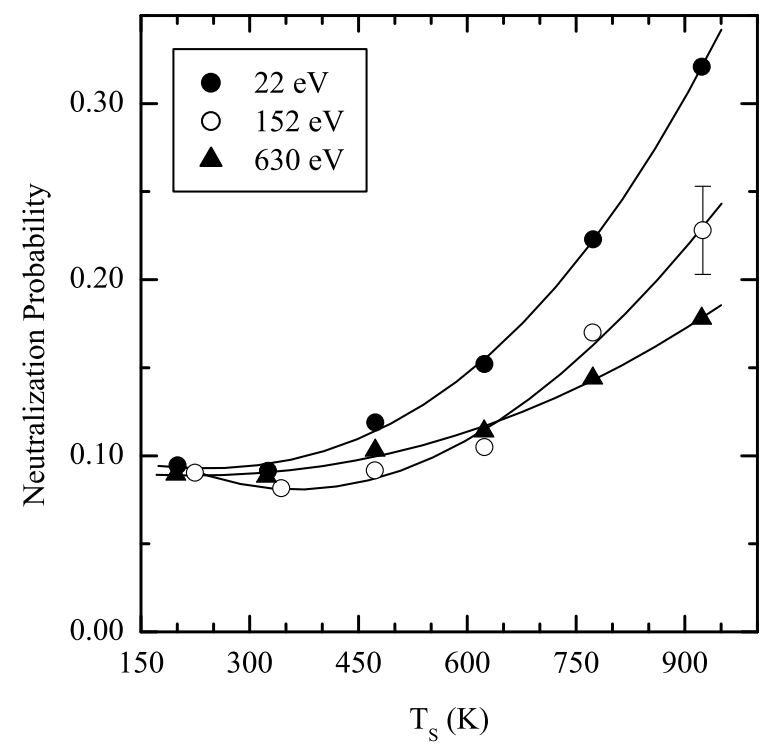

FIG. 2: Temperature-dependent neutralization results for $22 \mathrm{eV}, 152 \mathrm{eV}$, and $630 \mathrm{eV} \mathrm{Na}{ }^{+}$ions scattered from a $\mathrm{Cu}(001)$ crystal. The lines are drawn to guide the eye, and a typical error bar is shown.

charge states outside the surface, however, make this picture more complex as they imply that there is a non-zero probability of obtaining any allowed charge state at a distance $z$.

For the $\mathrm{Na}-\mathrm{Cu}(001)$ system, only the two charge states, neutral and positive, need to be considered. The probability of obtaining either charge state at a distance $z$ is determined primarily by the energetically favored charge state at that distance. Looking at Fig. \#, we see that this implies that the positive ion will dominate at most $z$ values. The velocity dependence at any $z$, however, is determined, to lowest order, by the relative magnitude of the projectile-surface coupling and the absolute energy difference between $I(z)$ and $E_{\mathrm{F}}$. Regions where either the coupling or this energy difference are largest determine the energyand coupling-dominated charge transfer regimes, respectively. If a projectile leaves the surface slowly, the energetically-favored charge state can be tracked to large distances, where the coupling is very small. In this case, the velocity dependence is classified as energydominated, and an exponential dependence of $P_{0}$ on the inverse perpendicular velocity is obtained [14]. Conversely, higher velocity projectiles only track the energetically-favored charge state near the surface where the coupling is large. This is the coupling-dominated regime where the simple exponential dependence of $P_{0}$ on inverse perpendicular velocity is no longer valid. 


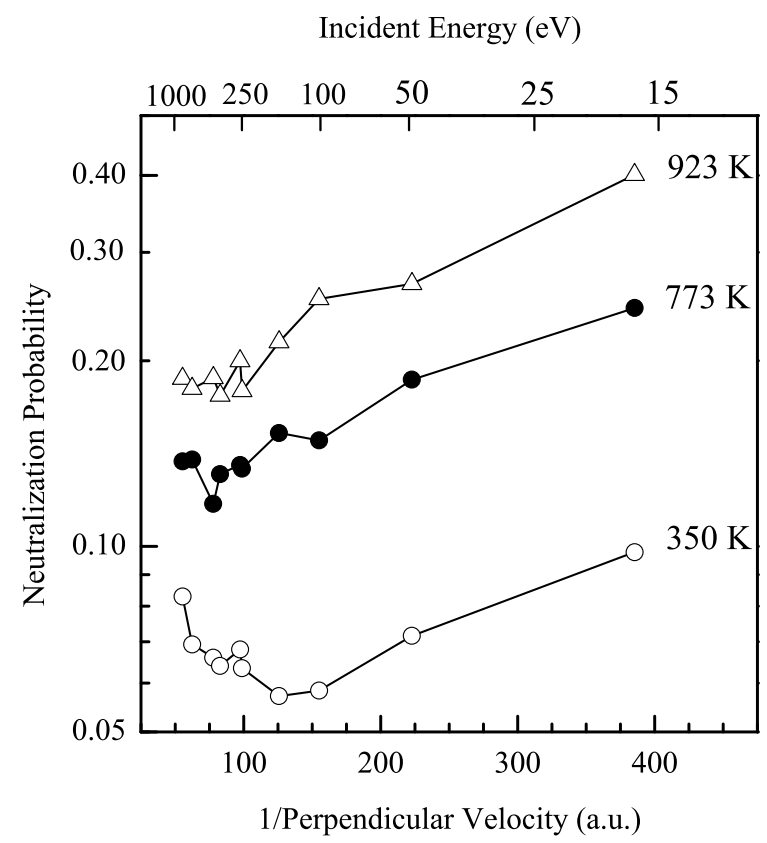

FIG. 3: The neutralization probability, $P_{0}$, for $\mathrm{Na}^{+}$ions scattered from a $\mathrm{Cu}(001)$ crystal at surface temperatures of $350 \mathrm{~K}, 773 \mathrm{~K}$, and $923 \mathrm{~K} . P_{0}$ is plotted on a logarithmic scale versus the inverse scattered perpendicular velocity to show the exponential dependence present at low energies and high temperatures. The velocity is shown in atomic units (a.u.), where 1 a.u. is approximately $2.2 \times 10^{8} \mathrm{~cm} / \mathrm{sec}$. The corresponding incident energy scale is noted along the top axis.

Focusing on the data taken at $350 \mathrm{~K}$ in Fig. 3, one can see evidence for both charge transfer regimes as a function of the inverse perpendicular velocity. Energy-dominated charge transfer occurs at the lowest velocities, giving $P_{0}$ an exponential dependence for incident energies less than $100 \mathrm{eV}$. Above $100 \mathrm{eV}$, the exponential dependence is no longer present, and $P_{0}$ increases. The strong projectile-surface interaction dominates at these higher velocities, giving an increased neutral occupancy, or as we measure it, a larger $P_{0}$ value [14]. Therefore, the non-monotonic change observed for $P_{0}$ indicates that both energyand coupling-dominated charge transfer occur in this system at $350 \mathrm{~K}$.

The data in Fig. 3 also show that the magnitude of $P_{0}$ and its dependence on inverse perpendicular velocity change dramatically with $T_{\mathrm{S}}$. Generally, an increase in $T_{\mathrm{S}}$ should alter the electron occupancy in the metal, populating metal levels above $E_{\mathrm{F}}$ according to the Fermi-Dirac distribution. If $\left|I(z)-E_{\mathrm{F}}\right| \lesssim k_{\mathrm{B}} T_{\mathrm{S}}$, thermal effects will become significant and lead to a larger $P_{0}$ value. Looking at Fig. 田, we see that this condition is met at large distances, where $I(z)$ crosses $E_{\mathrm{F}}$. This is the energy-dominated regime that is probed at 


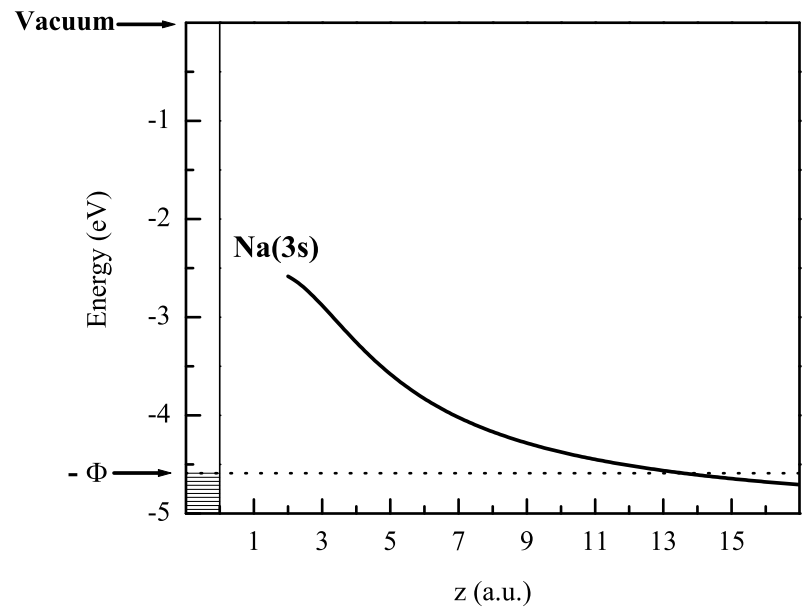

FIG. 4: An ionization level diagram for Na outside of a $\mathrm{Cu}(001)$ surface. The Fermi level lies at an energy $\Phi$ below the vacuum level, where $\Phi=4.59 \mathrm{eV}$ for $\mathrm{Cu}(001)$. Filled electron levels in the metal are indicated by the lines drawn on the far left. The distance, $z$, is plotted in atomic units (a.u.), where 1 a.u. is $0.529 \AA$.

low velocities, and it explains why the measured $P_{0}$ values are very sensitive to $T_{\mathrm{S}}$ as the scattered perpendicular velocity is decreased. At higher velocities, $I(z)$ is well above $E_{\mathrm{F}}$, the charge transfer is coupling-dominated, and consequently, the effects of $T_{\mathrm{S}}$ are reduced.

We have compared our measured temperature-dependent $P_{0}$ data to results obtained with an independent particle calculation[21]. This calculation is based on the spinless one-level Newns-Anderson Hamiltonian and allows for a determination of the occupancy of the $\mathrm{Na}$ atomic level after scattering from the $\mathrm{Cu}$ surface. It is a quantum mechanical treatment that correctly models both of the charge transfer regimes that are probed in our results. This is important because semi-classical treatments, such as the rate equations, do not reproduce the phenomena of coupling-dominated charge transfer that lead to the non-monotonic $P_{0}$ dependence observed in Fig. 3. A modified Fermi-Dirac distribution that incorporates both thermal and velocity-smearing effects was included in the calculation. The distribution was obtained by performing an angular average of a velocity-shifted Fermi-Dirac distribution over a spherical Fermi surface and is written as

$$
f^{*}(\epsilon)=\frac{\ln \left(1+\mathrm{e}^{-\beta(\epsilon-e)}\right)-\ln \left(1+\mathrm{e}^{-\beta(\epsilon+e)}\right)}{2 \beta e},
$$

where $\beta=1 / k_{\mathrm{B}} T_{\mathrm{S}}$ and $\epsilon$ is the energy of a metallic level relative to $E_{\mathrm{F}}$. The term $e$ is equal to $k_{\mathrm{F}} v_{\text {proj }}$, where $k_{\mathrm{F}}$ and $v_{\text {proj }}$ are the Fermi wavevector and the projectile velocity, 


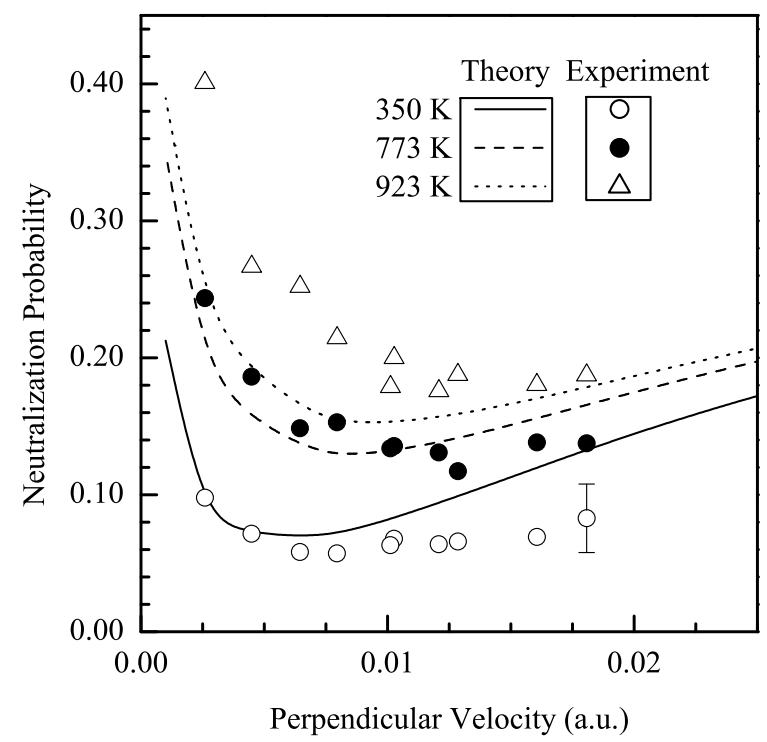

FIG. 5: Comparison of the experimental neutralization probability(symbols) to the results of the independent-particle calculation(lines) for $\mathrm{Na}^{+}$scattering from $\mathrm{Cu}(001)$. A typical error bar is shown.

respectively. The projectile-surface coupling used in the calculation was based on a fit to the theoretical results of Nordlander and Tully [22].

A comparison of our theoretical and experimental results for $P_{0}$ as a function of the perpendicular velocity at three different $T_{\mathrm{S}}$ values is shown in Fig. 5. The calculation reproduces the increase observed in $P_{0}$ with $T_{\mathrm{S}}$ as well as the non-monotonic change seen in $P_{0}$ with perpendicular velocity. Also, the large increase in $P_{0}$ observed at the lowest velocity values is reproduced. The quantitative differences evident between the experimental and calculation results shown in this figure vary with velocity and are very sensitive to the coupling and the inclusion of parallel velocity effects [23. Although a more complex neutralization model may be required to correctly deal with this interplay between $T_{\mathrm{S}}$, parallel velocity, and the projectile-surface coupling, it is remarkable that such a simple calculation can reproduce the observed trends.

In this Letter, we have shown that $T_{\mathrm{S}}$ can have significant effects on the $P_{0}$ values measured in hyperthermal energy ion-surface scattering experiments. By using $\mathrm{Na}-\mathrm{Cu}(001)$ as the experimental system, our results reveal that these thermal effects are present for both energy- and coupling-dominated charge transfer. The sensitivity of these alkali ion measurements to $T_{\mathrm{S}}$ in the energy-dominated regime demonstrate that the failure to include $T_{\mathrm{S}}$ 
in the analysis or modeling of neutralization experiments performed at finite temperatures could lead to large errors. Furthermore, in light of these results, future experiments aimed at measuring thermal effects due to many-body or Kondo effects for alkaline-earth projectiles [5, 6], must be carefully interpreted.

This work was supported by the National Science Foundation (NSF-DMR-9722771 and NSF-DMR-9712391). J.R.H. acknowledges support from a National Physical Science Consortium Fellowship.

* Corresponding author: sosolik@nist.gov; Present Address: National Institute of Standards and Technology, Gaithersburg, MD, 20899-8412

$\dagger$ Present Address: Woods Hole Oceanographic Institution, Woods Hole, MA 02543

$\ddagger$ Deceased.

[1] B.H. Cooper and E.R. Behringer, in Low Energy Ion-Surface Interactions, edited by J.W. Rabalais (J. Wiley \& Sons, New York, 1994).

[2] Here we refer to "charge transfer" as meaning the resonant transfer of charge between projectile and metal levels at the same energy.

[3] E.G. Overbosch, B. Rasser, A.D. Tenner, and J. Los, Surf. Sci. 92, 310(1980).

[4] Y. Bu, E.F. Greene, and D.K. Stewart, J. Chem. Phys. 92, 3899(1990).

[5] H. Shao, P. Nordlander, and D.C. Langreth, Phys. Rev. B 52, 2988(1995); Phys. Rev. Lett. 77, 948(1996).

[6] J. Merino and J.B. Marston, Phys. Rev. B 58, 6982(1998).

[7] B. Rasser and M. Remy, Surf. Sci. 93, 223(1980).

[8] R. Brako and D.M. Newns, Surf. Sci. 108, 253(1981); Vacuum 32, 39(1982).

[9] K.C. Liu, T.F. George, and K.-S. Lam, Solid State Commun. 53, 67(1985).

[10] F. Battaglia, K.C. Liu, and T.F. George, Int. J. Quantum Chem. Suppl. 19, 477(1986).

[11] S.I. Easa and A. Modinos, Surf. Sci. 183, 531(1987).

[12] H. Nakanishi, H. Kasai, and A. Okiji, Surf. Sci. 197, 515(1988).

[13] K.W. Sulston and F.O. Goodman, J. Chem. Phys. 112, 2486(2000); F.O. Goodman and K.W. Sulston, J. Chem. Phys. 114, 3265(2001).

[14] G.A. Kimmel and B.H. Cooper, Phys. Rev. B 48, 12164(1993). 
[15] R.L. McEachern et al., Rev. Sci. Instrum. 59, 2560 (1988).

[16] D.L. Adler and B.H. Cooper, Rev. Sci. Instrum. 59, 137 (1988).

[17] D.R. Peale, D.L. Adler, B.R. Litt, and B.H. Cooper, Rev. Sci. Instrum. 60, 730 (1989).

[18] G.A. Kimmel and B.H. Cooper, Rev. Sci. Instrum. 64, 672 (1993).

[19] All three peaks were used to determine $P_{0}$ as little variation was observed for the different trajectory types represented. The assigned velocity value was obtained by averaging over the velocities present in each spectrum.

[20] $I(z), I_{0}$, and $z$ are expressed here in atomic units.

[21] A.V. Onufriev and J.B. Marston, Phys. Rev. B 53, 13340(1996).

[22] P. Nordlander and J.C. Tully, Surf. Sci. 211/212, 207(1989).

[23] C.E. Sosolik, Ph.D. thesis, Cornell University, 2001. 